\section{Differentielle Streuraten \\ bei der Entstehung metastabiler Helium-Atome durch Elektronenstoß}

\section{G. Willems und R. Taubert}

Mitteilung aus der Physikalisch-Technischen Bundesanstalt Braunschweig

(Z. Naturforsch. 25 a, 449-451 [1970]; eingegangen am 5. Februar 1970)

Bei der experimentellen Untersuchung der Streuung von Elektronen an Atomen und Molekülen ist der direkte Nachweis der unter sehr großen Winkeln gestreuten Elektronen aus geometrisch-apparativen Gründen nicht möglich. Dagegen sind die aus einem Molekularstrahl durch Elektronenstoß herausgestreuten schweren Teilchen wegen der verhältnismäßig kleinen Streuwinkel im Laborsystem (L-System) in allen Fällen leicht zugänglich. Der Nachweis dieser Teilchen ist einfach, wenn es sich um metastabile Atome handelt. In einer Molekularstrahlapparatur ${ }^{1}$ wurde daher das folgende Experiment ausgeführt :

Ein Elektronenstrahl kreuzt einen gut kollimierten Helium-Atomstrahl unter einem Winkel von $90^{\circ}$. Die durch Elektronenstoß bei verschiedenen Elektronenenergien $e U_{\mathrm{e}}$ erzeugten metastabilen Atome werden mit einem in der Strahlebene um das Streuzentrum schwenkbaren Detektor [hier: Sekundärelektronenver- vielfacher (SEV) vom Kanaltyp] nachgewiesen. Die grundsätzlichen Schwierigkeiten und Grenzen dieser Methode lassen sich an Hand eines Newton-Diagramms, das den Zusammenhang zwischen den Geschwindigkeiten im Laborsystem und im Schwerpunktsystem herstellt (Abb. 1), übersehen. Da die Elektronengeschwindigkeit $v_{\mathrm{e}}$ viel größer ist als die Heliumgeschwindigkeit $v_{\mathrm{He}}$, verläuft die Relativgeschwindigkeit $\boldsymbol{v}_{\mathbf{r}}=\boldsymbol{v}_{\mathrm{He}}-\boldsymbol{v}_{\mathrm{e}}$ fast parallel zu $\boldsymbol{v}_{\mathrm{e}}$. Die Winkel- und Geschwindigkeitsverteilungen der Stoßpartner führen zu einer Vielzahl sich überlagernder Newton-Diagramme und damit zu einer Verringerung des Auflösungsvermögens. Der Einfluß der Geschwindigkeitsverteilungen im Heliumstrahl ist dabei dominierend. Diese Verteilung wurde nicht gemessen, sondern in Anlehnung an frühere Messungen der Verteilung eines Argon-Überschallstrahles mit einer Machschen Zahl Ma $\approx 7$ (relative Halbwertsbreite 27\%, wahrscheinlichste Geschwindigkeit $569 \mathrm{~ms}^{-1}$ ) abgeschätzt ${ }^{1}$.

Beim unelastischen Stoß der He-Atome mit den Elektronen büßen die letzteren einen der jeweiligen Anregungsenergie $E_{\mathrm{a}}$ entsprechenden Teil ihrer Energie im Schwerpunktsystem (S-System) ein. Der Impulssatz fordert eine korrespondierende Verringerung der $\mathrm{Ge}$ schwindigkeit $v_{\mathrm{He}, \mathrm{C}}^{\mathrm{f}}=v_{\mathrm{He}, \mathrm{C}}^{\mathrm{i}} \sqrt{1-\left(E_{\mathrm{a}} / e U_{\mathrm{e}}\right)}$ der angeregten He-Atome im S-System, läßt aber alle Flugrichtungen im S-System nach dem Stoß grundsätzlich

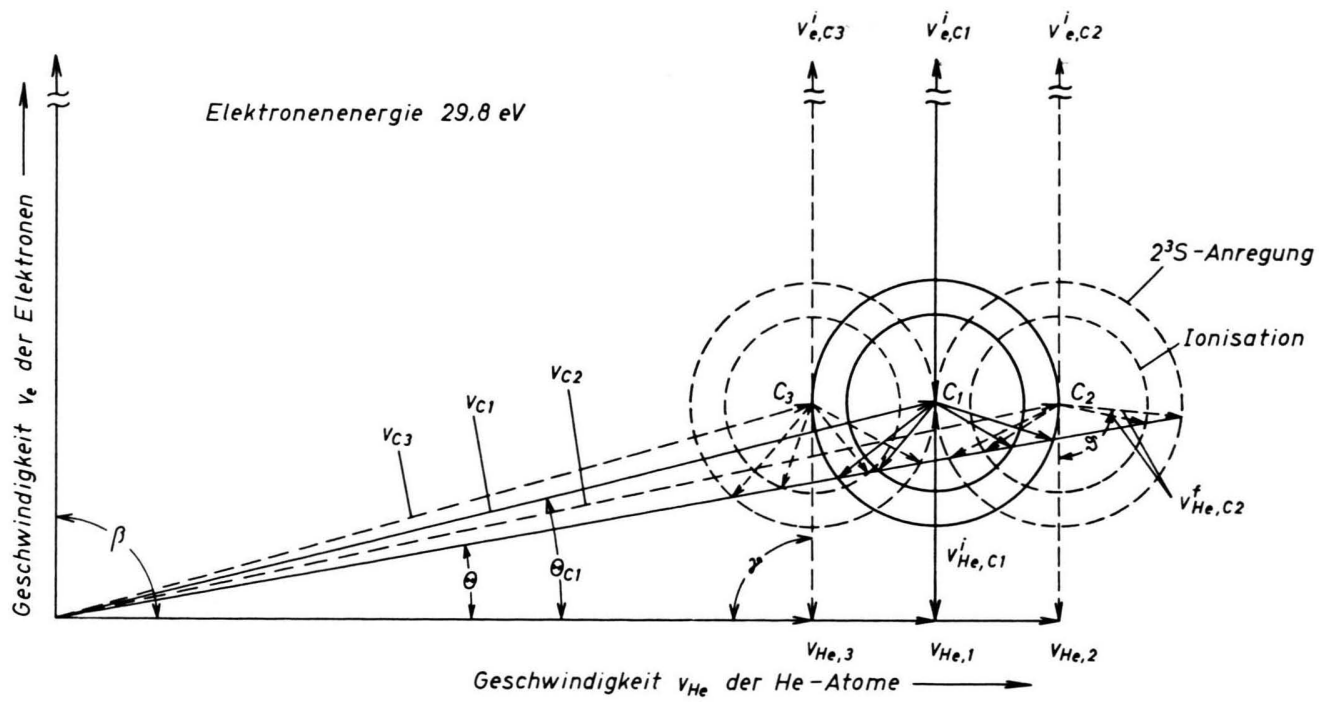

Abb. 1. Newton-Diagramm (Ausschnitt) zum Stoßprozeß $\mathrm{He}+\mathrm{e} \rightarrow \mathrm{He}^{*}+\mathrm{e}$ für drei Heliumgeschwindigkeiten $\left(v_{\mathrm{He}, 1}=\right.$ $1,80 \cdot 10^{5} \mathrm{~cm} \mathrm{~s}^{-1}, v_{\mathrm{He}, 2}=2,05 \cdot 10^{5} \mathrm{~cm} \mathrm{~s}^{-1}$ und $v_{\mathrm{He}, 3}=1,55$ $\cdot 10^{5} \mathrm{~cm} \mathrm{~s}^{-1}$ ) im L-System. $v_{\mathrm{e}}=$ Elektronengeschwindigkeit im L-System vor dem Stoß. $\boldsymbol{v}_{\mathrm{He}, \mathrm{C} j}^{1}$ und $\boldsymbol{v}_{\mathrm{e}, \mathrm{C} j}^{1}(j=1,2,3)$ sind die Helium- bzw. Elektronengeschwindigkeiten im Schwerpunkt-System (S-System) vor dem Stoß; $\boldsymbol{v}_{\mathrm{He}, \mathrm{C} j}^{\mathrm{t}}$ sind die Ge-

Sonderdruckanforderungen an die Physikalisch-Technische Bundesanstalt, Referat S, D-3300 Braunschweig, Bundesallee 100. schwindigkeiten der metastabilen He-Atome nach dem Stoß im S-System (nur zwei dieser Vektoren sind in Abb. 1 bezeichnet). $\Theta$ und $\vartheta$ sind die Streuwinkel im L- bzw. S-System, $\vartheta$ wurde nur einmal eingetragen. $C_{j}(j=1,2,3)$ sind die Schwerpunkte, $\boldsymbol{v}_{\mathrm{C}_{j}}$ die zu den eingetragenen Schwerpunkten gehörigen Schwerpunktgeschwindigkeiten. $\Theta_{\mathrm{C}_{j}}=$ Winkel zwischen $\boldsymbol{v}_{\mathrm{C}}$ und $\boldsymbol{v}_{\mathrm{He}}$.

1 N. Pachur, G. Willems u. R. Taubert, Z. angew. Phys. 28, 77 [1969]. 
$\mathrm{zu} \quad\left(v_{\mathrm{He}, \mathrm{c}}^{1} \mathrm{He}-\mathrm{Geschwindigkeit}\right.$ im S-System vor dem Stoß). Neben der direkten Anregung der mit dieser Meßanordnung nachweisbaren metastabilen Heliumzustände $\left(2^{3} \mathrm{~S}: E_{\mathrm{a}}=19,82 \mathrm{eV} ; 2^{1} \mathrm{~S}: E_{\mathrm{a}}=20,61 \mathrm{eV}\right)$ ist die Besetzung dieser Niveaus über Kaskadenprozesse mit Photonenemission möglich. In Abb. 1 sind die beiden Extremfälle - Anregung in den $2^{3}$ S-Zustand und Anregung in einen Zustand in der Nähe der Ionisierungsenergie - eingezeichnet.

Jedem Streuwinkel $\Theta$ im L-System entsprechen zwei Streuwinkel $\vartheta \mathrm{im}$ S-System. Damit ist zunächst eine eindeutige Umrechnung der L-Verteilung in die S-Verteilung ohne zusätzliche Informationen, die in der Geschwindigkeitsverteilung der gestreuten metastabilen He-Atome enthalten sind, nicht möglich.

Gemessen wurde die Winkelverteilung $\mathrm{d} I(\Theta) / \mathrm{d} \Omega$ der metastabilen He-Atome im L-System für verschiedene Werte $U_{\mathrm{e}}(\mathrm{d} \Omega$ Raumwinkelelement im L-System). Die Elektronenenergie wurde durch eine Appearancepotentialmessung an der Einsatzschwelle der MetastabilenProduktion ( $\left.2^{3} \mathrm{~S}-\mathrm{Zustnd}\right)$ kalibriert.

Die durch die metastabilen Atome im SEV erzeugten Ladungsimpulse wurden nach ihrer Verstärkung in einem ladungsempfindlichen System mit einem Zähler registriert. Ionen wurden durch einen Ablenkkondensator vom SEV ferngehalten; ein weiteres Plattenpaar erlaubte die optimale Ausrichtung des Elektronenstrahls zum Atomstrahl.

Abb. 2 zeigt einige Meßkurven. Die Meßpunkte wurden im allgemeinen in Schritten von $30^{\prime}$ aufgenommen, Parameter ist die Elektronenstoßenergie $e U_{\mathrm{e}}$. Die Streuung der Meßpunkte um die ausgezogenen Kurven ist vernachlässigbar, so daß darauf verzichtet wurde, die einzelnen Meßpunkte einzuzeichnen. Aus diesen Meßkurven läßt sich auf folgende Weise die differentielle Streurate $\mathrm{d} I(\vartheta) / \mathrm{d} \omega$ im S-System angenähert ermitteln: Nach bekannten Formeln ${ }^{2}$ werden bei einem Stoßwinkel $\beta=\pi / 2$ für Parametersätze $v_{\mathrm{e}}, v_{\mathrm{He}}, E_{\mathrm{a}}$ der Zusammenhang zwischen $\Theta$ und $\vartheta$ und die Raumwin. kelkorrektur $\mathrm{d} \omega / \mathrm{d} \Omega$ ( $\mathrm{d} \omega$ Raumwinkelelemente im SSystem) berechnet. Beispiele dafür gibt $\mathrm{Abb} .3$ für die bereits in Abb. 1 benutzten $v_{\mathrm{He}}$-Werte, $U_{\mathrm{e}}=29,8 \mathrm{~V}$ und $E_{\mathrm{a}}=20,61 \mathrm{~V}$. Die Raumwinkelkorrekturen ergeben für jeden Ast der $\vartheta=f(\Theta)$-Funktionen ein qualitativ gleiches Funktionsbild: ein fast rechteckiger Verlauf mit steilem Anstieg in den Bezirken streifenden Detektoreinblicks.

Für die weitere Auswertung wird vorausgesetzt, daß die Streuung zur Richtung der Relativgeschwindigkeit symmetrisch ist. Ferner beschränken wir uns darauf, im folgenden mit der wahrscheinlichsten He-Geschwindigkeit $v_{\mathrm{He}, 1} \mathrm{zu}$ rechnen.

Die Streurate in einen beliebigen Laborwinkel $\Theta_{1}$ (siehe Abb. 3) setzt sich zusammen aus den beiden Streuraten in die zugehörigen Winkel $\vartheta_{1}$ und $\vartheta_{2}$ des S-Systems, wobei die Raumwinkelkorrektur zu berücksichtigen ist. Gibt man die zu $\vartheta_{1}$ gehörige Streurate zu-

2 H. Pauly u. J. P. Toennies, in: Methods of Experimental Physics, Vol. 7, Part A. Edited by B. Bederson and W. L. Fite, Academic Press, N. Y., London 1968.

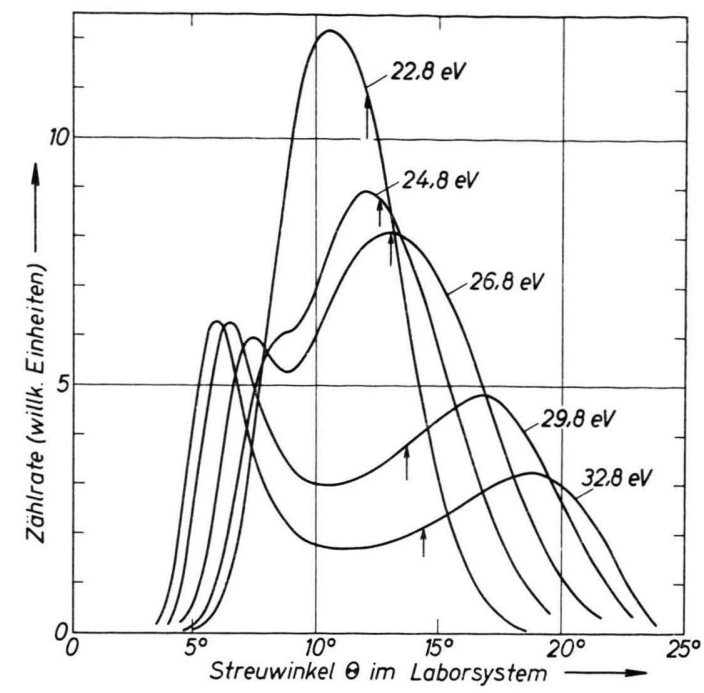

Abb. 2. Gemessene Streuraten, aufgetragen über dem Streuwinkel $\Theta$ im L-System. Kurvenparameter ist die Elektronen. energie $e U_{\mathrm{e}}$. Die Pfeile an den Kurven geben die jeweilige Lage von $\Theta_{\mathrm{C}}$ für $v_{\mathrm{He}, 1}=1,80 \cdot 10^{5} \mathrm{~cm} / \mathrm{s}$ an.

nächst willkürlich vor, so ist die Streurate in $\vartheta_{2}$ festgelegt. Der Streuwinkel $\vartheta_{2}-$ und damit die gerade festgelegte Streurate - gehört andererseits auch zu einem Laborwinkel $\Theta_{2}$. Daher ist auch die Streurate in den zweiten zu $\Theta_{2}$ gehörigen Streuwinkel $\vartheta_{3}$ festgelegt usw. Die Folge der $\vartheta_{1}, \vartheta_{2}, \vartheta_{3}, \ldots$ ist durch einen treppenförmigen Polygonzug zwischen den beiden Ästen der Funktion $\vartheta=f(\Theta)$ bestimmt. Die Wahl der 1. Streurate wird dadurch eingeengt, daß die daraus folgenden Streuraten im S-System nicht negativ werden dürfen und keine übermäßigen „Oszillationen“ zeigen sollten. Ebenso müssen die aus Polygonzügen mit verschie-

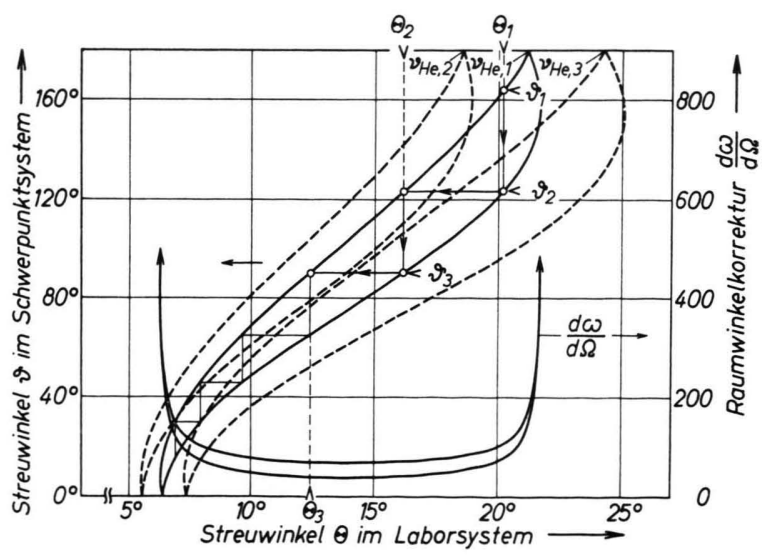

Abb. 3. Für $U_{\mathrm{e}}=29,8 \mathrm{~V}$ berechnete Abhängigkeit des Streuwinkels $\Theta$ im L-System vom Streuwinkel $\vartheta$ im S-System für die drei Heliumgeschwindigkeiten $v_{\mathrm{He}, j}(j=1,2,3)$. Es ist nur die Anregung in den $2^{1} \mathrm{~S}$-Zustand eingetragen. Weiterhin ist die berechnete Raumwinkelkorrektur $\mathrm{d} \omega / \mathrm{d} \Omega$ für $v_{\mathrm{He}, 1}$ eingetragen. Der Treppenzug verdeutlicht das Auswerteverfahren (siehe Text). 
denen Startwinkeln $\Theta_{1}$ gewonnenen Streuraten sich zu einem gemeinsamen Kurvenzug zusammensetzen (siehe Abb. 4). Die geringfügig "negative“ Streurate bei $\vartheta \approx 176^{\circ}$ wird dadurch verursacht, daß etwas oberhalb von $\Theta=20^{\circ}$ wegen der Geometrie der benutzten kommerziellen Elektronenstrahlquelle die gestreuten $\mathrm{He}$ Atome in zunehmendem Maße ausgeblendet werden.

Dieses summarische Auswerteverfahren berücksichtigt nicht die Verteilungsbreiten der verschiedenen Parameter, sondern ordnet die Meßkurven diskreten Parametersätzen zu. Die Verteilung des Parameters „Anregungsenergie“ ist, bedingt durch die möglichen Kaskadenprozesse, innerhalb zweier Schranken $\left(2^{3} \mathrm{~S}-\mathrm{Zu}\right.$ -

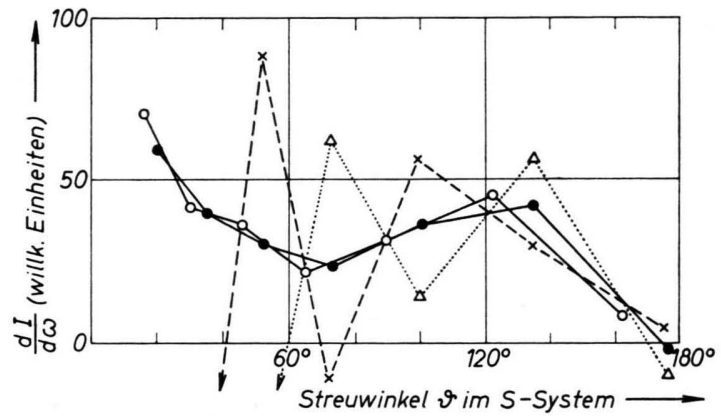

Abb. 4. $\mathrm{d} I / \mathrm{d} \omega$ aufgetragen über $\vartheta$; berechnet aus der gemessenen Kurve $\mathrm{d} I / \mathrm{d} \Theta$ für $U_{\mathrm{e}}=29,8 \mathrm{~V}$ und $v_{\mathrm{He}, 1}=1,8 \cdot 10^{5} \mathrm{~cm} \mathrm{~s}^{-1}$. Die Punktfolge $\bigcirc \circ \circ$ gehört zu dem in Abb. 3 eingezeichneten Polygonzug, die Punktfolgen $\bullet, X$ und $\triangle$ gehören zu einem anderen Polygonzug und unterscheiden sich durch die Vorgabe der Streurate bei $\vartheta=176^{\circ}$. Die Punktfolgen $\times$ und $\triangle$ sind offensichtlich zu verwerfen. stand und Ionisierungsgrenze) unbekannt. Bei der Auswertung wurde deshalb die direkte Anregung eines metastabilen Zustandes vorausgesetzt. Im Vergleich zu einer Elektronenenergie $e U_{\mathrm{e}}=29,8 \mathrm{~V}$, die den Abb. 3 und 4 zugrunde liegt, fällt der energetische Abstand der beiden metastabilen Zustände bei der $\vartheta \longleftrightarrow \Theta$. Zuordnung nur geringfügig ins Gewicht.

Für die Auswertung wurde willkürlich der $2^{1} \mathrm{~S} \mathrm{Zu}$ stand herangezogen. In Abb. 4 sind für die Heliumgeschwindigkeit $v_{\mathrm{He}, 1}$ und $U_{\mathrm{e}}=29,8 \mathrm{~V}$ die Auswerteergebnise für zwei verschiedene Polygonzüge dargestellt. Die stark oszillierenden Punktfolgen sind offensichtlich zu verwerfen. Dagegen ordnen sich die Punktfolgen $\bullet-$ und $\bigcirc \circ \circ$ in Abb. 4 zu einem glatten Kurvenverlauf, der auf einen erheblichen Streuanteil in den rückwärtigen Halbraum $(\vartheta>\pi / 2)$ mit einem flachen Maximum in der Umgebung von $\vartheta=120^{\circ}$ hinweist. Ein Ansteigen der Streurate bei Streuwinkeln $\vartheta>90^{\circ}$ ist bereits in Meßergebnissen von EHRHARDT, LANGHANS und LINDNER ${ }^{3}$ angedeutet. Die Messungen dieser Autoren erstrecken sich jedoch nur bis $\vartheta=110^{\circ}$.

Durch Verminderung der Energieunschärfe des Atomstrahls kann diese Versuchstechnik wesentlich verbessert werden. Dies kann entweder durch Einsatz eines Geschwindigkeitsselektors oder durch Erhöhung der Machschen Zahl der Überschallströmung geschehen.

Der Deutschen Forschungsgemeinschaft danken wir für die Mittel zum Bau der Streuapparatur.

3 H. Ehrhardt, L. Langhans u. F. Lindner, Z. Phys. 214, 179 [1968]. 